\title{
THE AUTOMORPHISM GROUP OF THE SEMIGROUP OF FINITE COMPLEXES OF A PERIODIC ABELIAN GROUP
}

\author{
R. D. BYRD, J. T. LLOYD and J. W. STEPP
}

(Received 22 May 1978)

Communicated by T. E. Hall

\begin{abstract}
In this paper it is shown that if $G$ is a periodic Abelian group and $|G| \geqslant 6$, then the only automorphisms of the semigroup of finite nonempty complexes of $G$ are induced by automorphisms of $G$.
\end{abstract}

Subject classification (Amer. Math. Soc. (MOS) 1970): primary 20 M 15; secondary 20 K 30.

Keywords and phrases: automorphism, periodic Abelian group, semigroup.

\section{Introduction}

In this paper we describe the automorphism group of the semigroup of finite nonempty complexes of a periodic abelian group (in terms of the automorphism group of the group). This work is a completion of the results by the current authors and Petersen (1977) in [BLPSa] where the automorphism group was described in the case that the group is periodic and locally cyclic.

Let $G$ be a group, written additively (but not necessarily abelian) and let $F(G)$ denote the collection of finite nonempty complexes (subsets) of $G$. Then $F(G)$ is a semigroup with respect to the operation $A+B=\{a+b \mid a \in A$ and $b \in B\}$. Mann $(1952,1953)$ investigated sums of sets of group elements. Recently more interest has been focused on various subsemigroups of the semigroup of all complexes of $G$. Trnkova (1975) proved that every abelian semigroup is embeddable in the semigroup of all complexes of $G$, for some abelian group $G$ and Lau (1979) gave a sufficient condition that a finite abelian semigroup could be embedded in the 
semigroup of all complexes of some finite abelian group. The semigroup $F(G)$ is central in the investigation of retractable groups presented by Byrd, Lloyd, Mena and Teller (1977). The automorphism group of $F(G)$ was studied for certain classes of groups in [BLPSa, $b$ and $c$ ].

The group of automorphisms of $F(G)$ will be denoted by Aut $F(G)$ and the group of automorphisms of $G$ will be denoted by Aut $G$. Each automorphism of $G$ induces an automorphism of $F(G)$ in a natural way. These elements of Aut $F(G)$ will be called standard automorphisms. It was shown in [BLPSc] that an element of Aut $F(G)$ is standard if and only if it preserves set inclusion. If $\alpha \in$ Aut $G$, then $\alpha^{*}$ will denote the standard automorphism of $F(G)$ induced by $\alpha$. The mapping which sends $\alpha$ to $\alpha^{*}$ is an isomorphism of $A u t F(G)$ onto the collection of standard automorphisms of $F(G)$.

It was also shown in [BLPSc] that if $G$ is a torsion-free abelian group, then $F(G)$ admits an infinite number of nonstandard automorphisms. In particular, it was shown that $\operatorname{Aut} F(Z)$ is countably infinite, where $Z$ denotes the additive group of integers, and it is well known that Aut $Z$ has only two elements. It was shown in [BLPSa] that if $Z_{n}$ denotes the cyclic group of order $n$, then the only $n$ 's for which $F\left(Z_{n}\right)$ admits nonstandard automorphisms are $n=3,4$ and 5. In [BLPSb] it was shown that the only periodic locally dihedral groups $D_{n}$ for which $F\left(D_{n}\right)$ admits nonstandard automorphisms is $D_{2}=Z_{2} \times Z_{2}$. In this paper we show that the only periodic abelian groups $G$ for which $F(G)$ admits nonstandard automorphisms are the ones mentioned above, namely $Z_{3}, Z_{4}, Z_{5}$ and $Z_{2} \times Z_{2}$.

We will identify $G$ with the group of units $\{\{g\} \mid g \in G\}$ of $F(G)$. If $X$ and $Y$ are sets, then $X \backslash Y$ denotes the elements in $X$ which do not belong to $Y$.

\section{Preliminaries}

Let $G$ be a group, $H$ be the subgroup of standard automorphisms of $F(G)$, and $K$ be the subgroup of automorphisms of $F(G)$ that are the identity on $G$ (the group of units of $F(G)$ ). Then $K$ is a normal subgroup of $A u t F(G), H \cap K=\{l\}$, and Aut $F(G)=K H$. Clearly, $F\left(Z_{2}\right)$ admits only standard automorphisms. The following information was given in [BLPSa], Section 3. If $G=Z_{3}$, then there exists $\theta \in$ Aut $F(G)$ with $\theta \mid G=\iota,\{0,1\} \theta=\{0,2\},\{0,1,2\} \theta=\{0,1,2\} ; K=\left\{\iota, \theta, \theta^{2}\right\}$, $H=\{\iota, \beta\}$, where $1 \beta=2$ and $\beta^{-1} \theta \beta=\theta^{2}$. Then Aut $F(G)=K H$ is isomorphic to $S_{3}$, the symmetric group of degree 3 . If $G=Z_{4}$, then there exists $\theta \in \operatorname{Aut} F(G)$ with

$$
\begin{aligned}
& \theta \mid G=\iota, \quad\{0,1\} \theta=\{0,3\}, \quad\{0,2\} \theta=\{0,2\}, \\
&\{0,1,2\} \theta=\{0,2,3\}, \quad\{0,1,2,3\} \theta=\{0,1,2,3\} ;
\end{aligned}
$$


$K=\left\{\iota, \theta, \theta^{2}, \theta^{3}\right\}, H=\{\imath, \beta\}$ where $1 \beta=3$ and $\beta^{-1} \theta \beta=\theta^{3}$. Then Aut $F(G)=K H$ is isomorphic to $D_{4}$, the dihedral group of order 8 . If $G=Z_{5}$, then there exists $\theta \in F(G)$ with $\{0, a\} \theta=\{2 a, 4 a\}$ for all $a \in G \backslash\{0\}$,

$$
\begin{aligned}
& \{0,1,2\} \theta=\{1,3,4\}, \quad\{0,1,3\} \theta=\{2,3,4\}, \\
& \{0,1,2,3\} \theta=\{0,1,2,3\}, \quad\{0,1,2,3,4\} \theta=\{0,1,2,3,4\} ;
\end{aligned}
$$

$K=\{\imath, \theta\}, H=\left\{\iota, \eta, \eta^{2}, \eta^{3}\right\}$, where $1 \eta=2$ and $\theta \eta=\eta \theta$. Then Aut $G=K H$ is isomorphic to $Z_{2} \times Z_{4}$. If $G=Z_{n}$ with $n \geqslant 6$, then $K=\{\imath\}$ and Aut $F(G)$ is isomorphic to Aut $G$. In [BLPSb] it was shown that if

$$
G=D_{2}=\langle a, b \mid 2 a=2 b, a+b=b+a\rangle,
$$

then there are three distinct automorphisms $\eta_{1}, \eta_{2}$ and $\eta_{3}$ of order 2 with $A \eta_{i}=A$ for all $A$ with $|A| \neq 3$ and

$$
\begin{aligned}
& \{0, a, b\} \eta_{1}=a+\{0, a, b\}, \\
& \{0, a, b\} \eta_{2}=b+\{0, a, b\}, \\
& \{0, a, b\} \eta_{3}=a+b+\{0, a, b\} .
\end{aligned}
$$

In this case $K=\left\{l, \eta_{1}, \eta_{2}, \eta_{3}\right\}$ and Aut $F(G)$ is isomorphic to $S_{4}$.

Lemma 1. Let $A, B \in F(G)$ with $|A|,|B|<|G|-1$ and $A \neq B$. Then there is $g \in G \backslash\{0\}$ with $A+\{0, g\} \neq B+\{0, g\}$.

PROOF. Let $x, y \in A \backslash B$ with $x \neq y$ and let $g=-y+x$. Then

$$
x \in(A+\{0, g\}) \backslash(B+\{0, g\}) .
$$

Hence, $A+\{0, g\} \neq B+\{0, g\}$.

The next two lemmas are from [BLPSa].

LeMmA 2. Let $\theta \in$ Aut $F(G)$ and let $H$ be a finite subgroup of $G$.

(i) Then $\theta \mid F(H)$ is a semigroup isomorphism of $F(H)$ onto $F(H \theta)$.

(ii) If $\theta \mid G=\imath$, then $H=H \theta$ and $\theta \mid F(H) \in \operatorname{Aut} F(H)$.

This is Lemma 3 of [BLPSa].

Lemma 3. If $A \in F(G)$ and $L(A)=\{g \mid g+A=A\}$, then $L(A)$ is a subgroup of $G$ and $A$ is a union of right cosets of $L(A)$. If $G$ is finite, then the number of translates of $A$ is the index of $L(A)$ in $G$. (If $g \in G$, then $g+A$ is $a$ translate of $A$.)

This is Lemma 7 of [BLPSa]. 
LEMMA 4. Let $G$ be a finite group such that $G=K \oplus\langle x\rangle$, for some subgroup $K$ and some $x \in G \backslash\{0\}$. If there exists $k \in K$ with $o(k)>2$, then there exist two element subsets $A_{1}, \ldots, A_{l}$ of $G$ so that

$$
G \backslash\{k\}=(K \backslash\{k\})+\sum_{n=1}^{l} A_{n} .
$$

Proof. Let $x$ and $k$ be given as above, $l=o(x)$ and $B_{0}=K \backslash\{k\}$. If

$$
B_{1}=B_{0}+(l-2)\{0, x\},
$$

then $k \notin B_{1}$. Let $B_{2}=B_{1}+\{0, x+k\}$. Then $G \backslash B_{2}=\{k, 2 k+(l-1) x\}$. Finally, let $B_{3}=B_{2}+\{0,-k+x\}=B_{2}+\{0,-(2 k+(l-1) x)+k\}$. Then $k \notin B_{3}$, and it remains to show that $2 k+(l-1) x \in B_{3}$. Now $2 k+(l-1) x=3 k+(l-2) x+(-k+x)$ which belongs to $B$ since $3 k \neq k$. Therefore,

$$
G \backslash\{k\}=B_{3}=(K \backslash\{k\})+(l-2)\{0, x\}+\{0, k+x\}+\{0,-k+x\}
$$

and the lemma is proven.

In order to prove the main result of this paper, it is necessary to show that $F\left(Z_{2} \times Z_{2} \times Z_{2}\right), F\left(Z_{3} \times Z_{3}\right), F\left(Z_{5} \times Z_{5}\right)$ and $F\left(Z_{2} \times Z_{2 p}\right)$, where $p$ is prime, admit only standard automorphisms. Since the proofs are similar, we do only Aut $F\left(Z_{3} \times Z_{3}\right)$.

Let $G=Z_{3} \times Z_{3}=\langle a, b \mid 3 a=3 b=0, a+b=b+a\rangle$ and let $\theta \in$ Aut $F(G)$ with $\theta \mid G=\iota$.

Claim 1. If $\{0, a\} \theta=\{0, a\}$, then $\{0, b\} \theta=\{0, b\}$.

Suppose (by way of contradiction) that $\{0, b\} \theta \neq\{0, b\}$. Since

$$
\theta \mid F(\langle b\rangle) \in \text { Aut } F(\langle b\rangle) \text {, }
$$

we have from the above that $\{0, b\} \theta=\{0,2 b\}$ or $\{0, b\} 0=\{b, 2 b\}$.

Case 1. $\{0, b\} \theta=\{0,2 b\}$. We consider the possible images of $\{0, a+b\}$.

Subcase 1.A. $\{0, a+b\} \theta=\{0, a+b\}$. Applying $\theta$ to both sides of the identity

$$
\{0, a, 2 a\}+\{0, a+b\}=\{0, a, 2 a\}+\{0, b\}
$$

we obtain

$$
\{0, a, 2 a\}+\{0, a+b\}=\{0, a, 2 a\}+\{0,2 b\},
$$

which is impossible. Hence, $\{0, a+b\} \theta \neq\{0, a+b\}$. 
Subcase 1.B. $\{0, a+b\} \theta=\{a+b, 2 a+2 b\}$. Again applying $\theta$ to both sides of the identity

$$
\{0, a, 2 a\}+\{0, a+b\}=\{0, a, 2 a\}+\{0, b\}
$$

we have that

$$
\{0, a, 2 a\}+\{a+b, 2 a+2 b\}=\{0, a, 2 a\}+\{0,2 b\}
$$

which is also impossible. Thus, $\{0, a+b\} \theta \neq\{a+b, 2 a+2 b\}$.

Subcase 1.C. $\{0, a+b\} \theta=\{0,2 a+2 b\}$. Then applying $\theta$ to both sides of the identity

$$
\{0, a\}+\{0, b, 2 b\}=\{0, a+b\}+\{0, b, 2 b\}
$$

we have that

$$
\{0, a\}+\{0, b, 2 b\}=\{0,2 a+2 b\}+\{0, b, 2 b\}
$$

which is again a contradiction. Therefore, $\{0, a+b\} \theta \neq\{0,2 a+2 b\}$. Consequently, we must have

Case 2. $\{0, b\} \theta=\{b, 2 b\}$. But this case yields a contradiction since if $\{0, b\} \theta=\{b, 2 b\}$, then $\{0, b\} \theta^{2}=\{0,2 b\}$, which was shown to be impossible by Case 1 . Thus, Claim 1 is established.

Claim 2. If $\{0, a\} \theta=\{0,2 a\}$ and $\{0, b\} \theta=\{0,2 b\}$, then $\{0, a+b\} \theta=\{0,2 a+2 b\}$.

Again we proceed by contradiction. By Claim 1, the only other possibility is $\{0, a+b\} \theta=\{a+b, 2 a+2 b\}$. Then, by applying $\theta$ to both sides of the identity

$$
\{0, a, 2 a\}+\{0, b\}=\{0, a, 2 a\}+\{0, a+b\},
$$

we obtain

$$
\{0, a, 2 a\}+\{0,2 b\}=\{0, a, 2 a\}+\{a+b, 2 a+2 b\},
$$

which is impossible. Thus, $\{0, a+b\} \theta=\{0,2 a+2 b\}$.

Claim 3. $\{0, g\} \theta=\{0, g\}$ for all $g$ in $G \backslash\{0\}$.

Suppose (by way of contradiction) there exists $g \in G \backslash\{0\}$ such that $\{0, g\} \theta \neq\{0, g\}$. By Claim 1 we may assume that there are $g, h \in G$ with $G=\langle g, h\rangle,\{0, g\} \theta=\{0,2 g\}$ and $\{0, h\} \theta=\{0,2 h\}$. By Claim 2,

$$
\{0, g+h\} \theta=\{0,2 g+2 h\} \text { and }\{0,2 g+h\} \theta=\{0, g+2 h\} \text {. }
$$

Hence, $\{0, g+2 h\} \theta=\{g+2 h, 2 g+h\}$. But using Claim 2 on the sets $\{0, h\}$ and $\{0, g+h\}$, we have that $\{0, g+2 h\} \theta=\{0,2 g+h\}$, which is a contradiction. 
Claim 4. $A \theta=A$ for all $A \in F(G)$.

If the assertion is not true, let $B \in F(G)$ be maximal with respect to $|B|$ and $B \theta \neq B$. By Claim 3 and Lemma $4,|B|<8$. If $L(B)=\{0\}$, then $|B+\{0, g\}|>|B|$ for all $g \in G \backslash\{0\}$ and so $B+\{0, g\}=(B+\{0, g\}) \theta=B \theta+\{0, g\}$ for all $g \in G \backslash\{0\}$. But, by Lemma $1, B=B \theta$, a contradiction. Thus, $L(B) \neq\{0\}$ and $B=L(B)+B_{1}$, where $\left|B_{1}\right| \leqslant 2$. But this implies that $B \theta=\left(L(B)+B_{1}\right) \theta=(L(B)) \theta+B_{1} \theta=L(B)+B_{1}$, again a contradiction. Therefore, $A \theta=A$ for all $A \in F(G)$. It now follows that Aut $\left(Z_{3} \times Z_{3}\right)$ consists only of standard automorphisms.

\section{Aut $F(G)$}

In this section we show that Aut $F(G)$ consists only of standard automorphisms if $G$ is a periodic abelian group with $|G| \geqslant 6$. The proof of the next lemma is straightforward and will be omitted.

LeMma 5. Let $G$ be a finite group, $H$ be a normal subgroup of $G, \eta$ the natural mapping of $G$ onto $G / H$, and $\theta \in A$ A $F(G)$ with $H \theta=H$. If $X \theta^{\#}=X \eta^{-1} \theta \eta$ for every $X \in F(G / H)$, then $\theta^{*} \in F(G / H)$. Moreover, if $A, B \in F(G)$ with $H \subseteq L(A)=L(B)$ and $A \theta \neq B$, then $A \eta \theta^{\#} \neq B \eta$.

THEOREM 1. Let $G$ be a finite abelian group with $|G| \geqslant 6$. Then Aut $F(G)$ consists only of standard automorphisms.

Proof. Assume that this is not the case. Let $G$ be a group which is minimal with respect to $|G| \geqslant 6$ and there is $B \in F(G), \theta \in \operatorname{Aut} F(G)$ with $\theta \mid G=\imath$, and $B \theta \neq B$.

Case 1. $G$ is isomorphic to the direct product of $n$ copies of $Z_{2}$, where $n>3$.

\section{Let}

$$
\left.G=\left\langle a_{1}, \ldots, a_{n}\right| 2 a_{i}=0 \text { for } i \in\{1, \ldots, n\} \text { and } a_{i}+a_{j}=a_{j}+a_{i} \text { for } i, j \in\{1, \ldots, n\}\right\rangle \text {. }
$$

It was pointed out in Section 2 that if $a, b, c \in G$ and $\langle a, b, c\rangle$ is isomorphic to $Z_{2} \times Z_{2} \times Z_{2}$, then $\theta \mid\langle a, b, c\rangle$ is a standard automorphism. Thus, $A \theta=A$ if $|A| \leqslant 3$. Since

$$
A=\left\{0, a_{1}, a_{2}\right\}+\left\{0, a_{1}, a_{3}\right\}+\ldots+\left\{0, a_{1}, a_{n}\right\}=G \backslash\left\{\sum_{i=1}^{n} a_{i}\right\},
$$

$A \theta=A$ if $|A| \geqslant|G|-1$. Now let $B \in F(G)$ be maximal with respect to $|B|$ and $B \theta \neq B$. Then $L(B) \neq\{0\}$, for otherwise we would have $|B|<|B+\{0, g\}|$ for all 
$g \in G \backslash\{0\}$ and $B+\{0, g\}=(B+\{0, g\}) \theta=B \theta+\{0, g\}$. Thus, by Lemma $1, B=B \theta$. Let $H$ be a subgroup of $L(B)$ with $|H|=2$. Then $\theta^{\#} \in$ Aut $F(G / H)$, $\theta^{\#}$ is not standard, and $|G / H| \geqslant 6$ and this contradicts the choice of $G$ as a minimal counterexample.

Case 2. There exists $x$ in $G$ with $o(x) \neq 2$.

Let $g \in G$. If $o(g)=2$, then $\{0, g\}$ is a subgroup and thus $\{0, g\} \theta=\{0, g\}$. If $o(g)>2$, then either $g$ is in a cyclic group of order larger than 5 or $g$ is in a subgroup isomorphic to $Z_{2} \times Z_{4}$. In either case we must have that $\{0, g\} \theta=\{0, g\}$. Therefore, $A \theta=A$ if $|A| \leqslant 2$ and by Lemma $4, A \theta=A$ if $|A| \geqslant|G|-1$. Now let $B \in F(G)$ be maximal with respect to $|B|$ and $B \theta \neq B$. As in Case $1, L(B) \neq\{0\}$. So let $H$ be a subgroup of $L(B)$ with $|H|$ a prime. Then, by Lemma 4 , Aut $F(G / H)$ admits non-standard automorphisms. By the minimality of $|G|$, we must have $G / H$ is isomorphic to $Z_{3}, Z_{4}, Z_{2} \times Z_{2}$ or $Z_{5}$.

Case 2.1. $G / H$ is isomorphic to $Z_{3}$.

In this case $G$ must be cyclic of order greater than 5 or $Z_{3} \times Z_{3}$. But both imply that Aut $F(G)$ consists only of standard automorphisms.

Case 2.2. $G / H$ is isomorphic to $Z_{4}$.

Again $G$ is either cyclic or $Z_{2} \times Z_{4}$. But both of these imply that Aut $F(G)$ consists of only standard automorphisms.

Case 2.3. $G / H$ is isomorphic to $Z_{2} \times Z_{2}$.

Then either $G$ is $Z_{2} \times Z_{2 p}$, where $p$ is prime, or $Z_{2} \times Z_{2} \times Z_{2}$. But again both of these imply Aut $F(G)$ consists only of standard automorphisms.

Case 2.4. $G / H$ is isomorphic to $Z_{5}$.

Then $G$ is cyclic or $Z_{5} \times Z_{5}$ and the same contradiction is obtained.

Thus it must be the case that Aut $F(G)$ admits only standard automorphisms if $G$ is a finite abelian group with $|G|>5$.

COROLLARY. If $G$ is a periodic abelian group with $|G| \geqslant 6$, then Aut $F(G)$ consists only of standard automorphisms. 


\section{References}

R. D. Byrd, J. T. Lloyd, R. A. Mena and J. R. Teller (1977), "Retractable groups", Acta Math. Acad. Sci. Hungar. 29, 219-233.

R. D. Byrd, J. T. Lloyd, F. D. Pedersen and J. W. Stepp (1977), "Automorphisms of the semigroup of finite complexes of a periodic locally cyclic group", Pacific J. Math. 72, 27-39. (BLPSa)

R. D. Byrd, J. T. Lloyd, F. D. Pederson and J. W. Stepp (1978), "Automorphisms of the semigroup of finite complexes of a locally finite group", J. Reine Angew. Math. 299/300, 151-160. (BLPSb)

R. D. Byrd, J. T. Lloyd, F. D. Pederson and J. W. Stepp (1979), "Automorphisms of the semigroup of finite complexes of a torsion-free abelian group" (submitted). (BLPSc)

A. Lau (1979), "Finite abelian semigroups represented into the power set of finite groups" (submitted).

H. B. Mann (1952), "On products of sets of group elements", Canad. J. Math. 4, 64-66.

H. B. Mann (1953), "An addition theorem for sets of elements of an abelian group", Proc. Amer. Math. Soc. 4, 423.

V. Trnkova (1975), "On a representation of commutative semigroups", Semigroup Forum 10, 203-214.

Department of Mathematics,

University of Houston,

Houston, Texas 77004,

USA. 